\title{
Implementación de la Educación Afectiva y Sexual. Un estudio exploratorio en tres escuelas secundarias públicas de Barcelona
}

\author{
Maria-Carmelita Lapadula ${ }^{1}$ \\ Artículo de estudios y tendencias. Recibido: 19/10/2020. Aceptado: 30/11/2020. Publicación avanzada: 16/12/2020. Publicado: 04/01/2021.
}

\section{Resumen}

INTRODUCCIÓN. El modelo de sexualidad integral, que abarca aspectos biológicos, sociales, psicológicos y culturales, define un modelo de educación sexual que propone una formación graduada, a lo largo de toda la vida, abordada desde diferentes servicios. La escuela, caracterizada por ser uno de los espacios primarios de socialización, es clave para el desarrollo de capacidades relacionadas con la sexualidad y la afectividad.

MÉTODO. Se propuso un estudio exploratorio que permitiera analizar cómo es percibida la implementación de la educación sexual en tres escuelas públicas de la ciudad de Barcelona. Para llevar a cabo la recogida de datos se realizaron entrevistas a estudiantes y docentes de 3. y 4. curso de Educación Secundaria Obligatoria de los tres centros estudiados.

RESULTADOS. Los resultados se recogieron en cuatro bloques, según la información obtenida: a) conceptualización de la Educación Sexual y Afectiva, b) acciones en la escuela, c) necesidades de los estudiantes y d) propuestas de mejora.

DISCUSIÓN. Finalmente se contrastaron los resultados obtenidos con estudios que trabajan sobre la necesidad de una educación sexual integral y transversal que garantice el ejercicio de la juventud.

Palabras clave

Educación sexual; Educación afectiva y sexual; Sexualidad; Adolescencia.

Datos de la autora

${ }^{1}$ Universitat de Barcelona, España. Máster en Intervenciones Socioeducativas. Investigadora predoctoral GRISIJ. Departamento de Métodos de Investigación y Diagnóstico en Educación. Contacto para la correspondencia: carmelita.lapadula@ub.edu

\section{Referencia recomendada}

Lapadula, M. C. (2021). Implementación de la Educación Afectiva y Sexual. Un estudio exploratorio en tres escuelas secundarias públicas de Barcelona. REIRE Revista d'Innovació i Recerca en Educació, 14(1), 1-17. https://doi.org/10.1344/reire2021.14.132595

(C) 2021 La autora. Este artículo es de acceso abierto sujeto a la licencia Reconocimiento 4.0 Internacional de Creative Commons, la cual permite utilizar, distribuir y reproducir por cualquier medio sin restricciones siempre que se cite adecuadamente la obra original. Para ver una copia de esta licencia, visite https://creativecommons.org/licenses/by/4.0/ 


\section{Títol (català)}

Educació afectiva i sexual. Un estudi exploratori en tres escoles públiques de Barcelona

\section{Resum}

INTRODUCCIÓ. El model de sexualitat integral, que engloba aspectes biològics, socials, psicològics i culturals, defineix un model d'educació sexual que proposa una formació graduada, durant tota la vida i abordada des de diferents serveis. L'escola, com un dels espais primaris de socialització, és clau per al desenvolupament de les capacitats relacionades amb la sexualitat i l’afectivitat.

MÈTODE. Es va proposar un estudi exploratori que permetés entendre com es percep la implementació de l'educació sexual en tres escoles públiques de la ciutat de Barcelona. La recollida de dades es va fer a través d'entrevistes a estudiants i docents de $3 r$ i 4 t curs d'educació secundària obligatòria.

RESULTATS. Els resultats es van recollir en quatre blocs, segons la informació obtinguda: $a$ ) conceptualització de l'educació sexual i afectiva, $b$ ) accions a l'escola, $c$ ) necessitats de l'alumnat i $d$ ) propostes de millora.

DISCUSIÓ. Els resultats es van contrastar amb estudis que treballen sobre la necessitat d'una educació sexual integral i transversal que garanteixi l'exercici dels drets dels i les joves.

Paraules clau

Educació sexual integral; Educació afectiva i sexual; Sexualitat; Adolescència.

\section{Title (English)}

Education in human affectivity and sexuality. An exploratory study at three publicly-funded schools in Barcelona

\section{Abstract}

INTRODUCTION. The comprehensive model of sexuality, which includes biological, social, psychological and cultural aspects, defines a model of sex education that proposes graded contents presented at different ages and by different services. The school, as a primary setting of socialization, has a vital role to play in the development of capacities related to sexuality and affectivity.

METHOD. This exploratory study aims to assess how sex education is taught at three publicly-funded schools in Barcelona. Data were collected through interviews with pupils in the third and fourth years of secondary school and their teachers.

RESULTS. The results were collected in four headings: a) the concept of education in affectivity and sexuality, b) interventions in the school, c) students' needs, d) proposals.

DISCUSSION. The results were contrasted with those of previous studies regarding the need for comprehensive and cross-sectional sex education to ensure young people's ability to exercise of their rights.

\section{Keywords}

Sex education; Sexuality education; Sexuality; Adolescence. 


\section{Introducción}

El currículo de educación sexual se ha ido modificando muy deprisa a lo largo de las últimas décadas, en consecuencia sobre todo a los cambios en la concepción de la sexualidad. En la educación media, conviven actualmente diferentes modelos, más o menos adaptados a los lineamientos de los organismos internacionales que marcan la agenda en materia de Derechos Sexuales y Reproductivos.

La falta de una ley específica que regule en España los contenidos y marque los lineamientos curriculares dificulta esta tarea (Companys, 2016), y puede agravar las desigualdades entre los estudiantes de diferentes escuelas, según los recursos simbólicos y materiales de los que disponen. Estas desigualdades se acrecientan en aquellos colectivos que se encuentran en mayor riesgo de exclusión social (Mateos et al., 2014).

Antes de comenzar, se comparten las consideraciones hechas en relación con los términos principales utilizados en la redacción de este artículo: «Educación sexual» se utiliza para hablar en general de todos los modelos considerados, pero especialmente de aquellos que no se corresponden con la concepción integral de sexualidad; "Educación Integral en Sexualidad» (EIS) corresponde al modelo actual propuesto por la Organización de las Naciones Unidas para la Educación, la Ciencia y la Cultura (UNESCO, 2018a), que supone una visión integral de la sexualidad y un modelo de educación y salud que abarca desde la niñez hasta la vejez; "Educación Sexual Integral» también responde a una propuesta integral, y es utilizado en la bibliografía y legislación latinoamericana, incluso anteriores al año 2018; "Educación Afectiva y Sexual» (EAS) es el término utilizado en la literatura española y catalana, así como en el marco normativo. Lo utilizaremos en este trabajo cuando hablemos específicamente del contexto en el cual se desarrolla este estudio.

La sexualidad es parte constitutiva del ser humano, que está presente durante todas las etapas de su vida y se expresa de diferentes maneras: el sexo, la identidad sexual, los roles de género, el erotismo, el placer, la intimidad, la reproducción y la orientación sexual (Organización Mundial de la Salud, 2006). Las luchas políticas de los movimientos feministas y de diversidad de género, han puesto a la sexualidad en el centro de la agenda política (Direcció de Feminismes i LGTBI, 2017; Garaizabal, 2009) lo que ha permitido llegar a una definición que integra diferentes aspectos sociales, psicológicos y de la salud de las personas.

Históricamente, la sexualidad estuvo ligada a la salud física y se trabajó desde un punto de vista biologicista, relacionado también a los antiguos modelos de medicina. Autoras y autores como Foucault (1977), Weeks (1981) o Cartledge y Ryan (1983) contribuyeron, en la segunda mitad del s. xx en la construcción de una definición de sexualidad que se alejara del punto de vista de las ciencias positivistas y considerara las variables históricas, culturales y sociales (Zemaitis, 2016).

Los modelos de educación sexual están ligados directamente a las diferentes concepciones de sexualidad, que aún coexisten en las instituciones y los servicios. Los estudios de Kirby (1980) y sus colaboraciones con la UNESCO (2015) permitieron adaptar las orientaciones internacionales para desarrollar los currículos escolares y de educación no formal en concordancia con las recomendaciones de la OMS (2018) en términos de promoción y prevención de la salud sexual.

En este sentido, podemos decir que contamos hoy con una variedad de recursos que nos permiten trabajar la Educación Integral en Sexualidad, entendida como una herramienta para el ejercicio de los derechos de todas las personas, contribuyendo a su desarrollo, de forma transversal, en las diferentes etapas de la vida. 
Implementación de la Educación Afectiva y Sexual. Un estudio exploratorio en tres escuelas secundarias públicas de Barcelona

Para esto es necesario implementarla, de manera adaptada, en los diferentes niveles y espacios educativos, servicios sociales y de salud. (Font et al., 2006; Jané et al., 2014).

La Educación Integral en Sexualidad, para garantizar los derechos de todas las personas, debe basarse en los principios fundamentales presentados por la UNESCO (2018a):

- Precisión científica

- Graduación

- Adecuación a cada edad y etapa de desarrollo

- Base en el currículum educativo

- Integralidad

- Enfoque de Derechos Humanos

- Promoción de la igualdad de género

- Relevancia cultural y contextual

- Capacidad transformativa

- Capacidad para desarrollar aptitudes necesarias que apoyen elecciones saludables

Teniendo en cuentas estas bases, apuntamos a un modelo de educación sexual con perspectiva de género, superadora del coitocentrismo y de la patologización de las diferencias, que permita la libertad de decisión sobre el propio cuerpo, así como el disfrute y la expresión de la sexualidad (Calvo et al., 2018).

Cuando hablamos de educación integral en sexualidad en la escuela, la pensamos como uno de los espacios primarios de socialización (Zemaitis, 2016). Por esta razón, surge la necesidad de contar con un currículo común que permita el acceso de todas y todos los estudiantes a la misma información.

Actualmente, coexisten diferentes modelos de educación sexual. En este sentido, es importante señalar algunas evidencias de la efectividad del modelo Integral en Sexualidad aplicado en la escuela, frente a otras propuestas:

- Los programas basados en este modelo han logrado reducir índices de embarazo adolescente y enfermedades de trasmisión sexual, retardar el inicio de las relaciones sexuales y disminuir las situaciones violentas y de riesgo (Kirby, 2007; UNESCO, 2018b).

- Los programas basados en la participación de familias, docentes, personal de los servicios de salud y otros actores aumentan su efectividad (Direcció de Feminismes i LGTBI, 2017; Generelo, 2016).

Por otra parte, los modelos que no apuntan a la integralidad, sino que se enfocan en prácticas o problemáticas específicas, y de manera puramente preventiva, han demostrado ser poco efectivos. Por ejemplo, los enfoques de abstinencia, que promueven la abstinencia hasta el matrimonio, o los de abstinencia-plus, que reconocen que existen prácticas sexuales anteriores al matrimonio y trabajan en la promoción del uso de preservativos, pueden influir en la creación de una concepción negativa de la sexualidad (Kirby, 2007). 
Implementación de la Educación Afectiva y Sexual. Un estudio exploratorio en tres escuelas secundarias públicas de Barcelona

En los últimos años se han desarrollado programas con este enfoque dirigidos a la adolescencia (Font et al., 2006; Jané et al., 2014; UNESCO, 2018a), etapa decisiva en el desarrollo de la salud, y durante la cual la sexualidad se vive de manera muy intensa (Jané et al., 2014):

En la adolescencia, cuando se comienza a tener la capacidad de pensar de forma hipotética y abstracta, se comienza a abandonar el pensamiento simplista y concreto por visiones de futuro más complejas [...] Es importante entender el pensamiento del adolescente, ya que, por primera vez, comienza a tomar decisiones. (Mateos, 2014, p. 10)

Mateos (2014) también señala que esta es la etapa en la cual comienzan a mantener relaciones sexuales y de pareja. Mientras tanto, quedan expuestos a situaciones de vulnerabilidad provocados por la gran cantidad de información errónea y confusa que reciben (UNESCO, 2018b). El acceso a la EIS permite la "adquisición y/o el fortalecimiento de valores como reciprocidad, igualdad, responsabilidad y respeto, prerrequisitos para relaciones sociales y sexuales más sanas y seguras" (UNESCO, 2010, p. 5), mientras que fortalece la construcción de la identidad (Consejería de Educación y Ciencia \& Instituto Andaluz de la Mujer, 1999; UNESCO, 2014) y disminuye las posibles situaciones de violencia de género (Direcció de Feminismes i LGTBI, 2017; Font et al., 2006; UNESCO, 2018b).

Es importante señalar que, a pesar de trabajar sobre un modelo con la escuela en el centro, la implementación de la EIS es un eje transversal del trabajo con las familias, los servicios de salud, sociales y otros espacios de educación no formal, entre otros actores comunitarios, incentivando el trabajo interdisciplinario (UNESCO, 2010).

Para cumplir con estos objetivos y promover "una educación basada en los derechos humanos, la igualdad de género, las relaciones, la reproducción, el comportamiento sexual de riesgo y la prevención de enfermedades desde una perspectiva positiva" (UNESCO, 2018b, párr. 7), la UNESCO (2018a) desarrolla unas Orientaciones Técnicas a partir de las cuales organizar un currículo teniendo en cuenta los diferentes niveles y condiciones simbólicas del estudiantado en los distintos contextos. De esta manera, se propone un punto de partida para trabajar la EIS en la escuela secundaria obligatoria, como un derecho básico, contemplado en la Declaración de los Derechos Sexuales y Reproductivos (Plataforma de Acción de Beijing, 1995). Según explica Aldavert (2019), los Derechos Sexuales y Reproductivos, en tanto que Derechos Humanos, son imprescindibles para alcanzar una vida digna. Las sociedades no pueden ser justas sin el reconocimiento, la garantía y el pleno ejercicio de estos derechos.

La necesidad de trabajar estas temáticas se hace patente al revisar la situación de las y los adolescentes en el estado español:

- El uso del preservativo entre los jóvenes ha disminuido del $84 \%$ al $75 \%$ en los últimos 16 años según el Ministerio de Sanidad (Agencia EFE, 2019)

- Las y los más afectados por las enfermedades de transmisión sexual son jóvenes entre 20 y 24 años ${ }^{1}$ (Agencia EFE, 2019)

- En 2017, el $10 \%$ de las personas condenadas por delitos cometidos contra la libertad e indemnidad sexuales fueron varones menores de 18 años (Instituto Nacional de Estadística, 2018)

${ }^{1}$ Edad cercana a la media del inicio de las relaciones sexuales coitales en España (Instituto Nacional de Estadística, 2003) 
Implementación de la Educación Afectiva y Sexual. Un estudio exploratorio en tres escuelas secundarias públicas de

- En 2016 se registraron 653 mujeres viviendo bajo protección contra la violencia machista (Agencia EFE, 2018).

- El mayor porcentaje de víctimas de violencias o incidentes de odio debido a su orientación sexual o identidad de género está entre las y los jóvenes de 12 a 35 años, según la FELGBT (Rebollo et al., 2019).

En este contexto, introducimos el marco normativo, así como el tipo de intervenciones y los programas vigentes en la ciudad de Barcelona.

Para comenzar a delinear el contexto de investigación es preciso definir que las escuelas públicas en Barcelona trabajan sobre un modelo de coeducación, que apunta a la transmisión del conocimiento y los procesos de enseñanza-aprendizaje a partir de la idea de igualdad entre las personas (Observatorio para la Igualdad de Oportunidades, 2008) y la reducción de las desigualdades.

Coexisten actualmente diferentes formas de aproximarse a la educación sexual. Companys (2016), por un lado, reconoce dos enfoques pedagógicos diferentes: un enfoque médico-preventivo, que reproduce perspectivas heteronormativas como el binarismo sexual y de género, y por otro lado un enfoque integral, que contempla una forma de trabajar transversal e interdisciplinaria, haciendo hincapié en la perspectiva de género y el reconocimiento de la diversidad. Este último modelo tiene como principal objetivo la promoción de la salud sexual en todos sus aspectos (BZgA. Federal Centre for Health and Education, 2010).

La coexistencia de estos dos modelos dificulta la igualdad de oportunidades entre el alumnado joven escolarizado, sumado a las dificultades que se producen en su posible implementación, según los recursos simbólicos y materiales de cada institución. Con esto nos referimos a las diferentes formas de intervención que se llevan a cabo, muchas veces ligadas a la capacidad de acceso a los recursos disponibles (SidaStudi, 2018). Estas formas de intervención pueden ser externas: formadores de otras instituciones que organizan talleres y actividades puntuales en los centros educativos; intervenciones mixtas: en las cuales esas actividades se combinan con trabajo de profesores o tutores sobre temas específicos; e intervenciones transversales, aplicadas en centros que trabajan la sexualidad y la afectividad desde el proyecto pedagógico (Companys, 2016; SidaStudi, 2018).

A falta de una ley específica, las intervenciones que se llevan adelante en el contexto de investigación se apoyan en legislaciones a nivel europeo, nacional y autonómico que brindan algunas herramientas para facilitar su acceso.

Entre ellas se encuentran la Resolución del Parlamento Europeo sobre salud sexual y reproductiva y los derechos en esta materia (Resolución del Parlamento Europeo sobre salud sexual y reproductiva y los derechos en esta materia 2001/2128(INI), 2003), que resalta la necesidad de legislaciones que contemplen la salud sexual y reproductiva desde una perspectiva global y positiva y propone un modelo de educación sexual que refuerce los existentes servicios de planificación familiar y sea participativa y adecuada para cada edad, haciendo especial énfasis en la juventud.

A nivel estatal, la Ley orgánica 2/2010 (Ley de salud sexual y reproductiva y de la interrupción del embarazo 2/2010, de 3 de marzo, 2010) es uno de los pilares que sostiene el derecho a la información y la comunicación en materia de sexualidad, apuntando a políticas de prevención del embarazo, así como de promoción de la salud sexual y el acceso a una educación afectiva y sexual en el sistema educativo. 
Implementación de la Educación Afectiva y Sexual. Un estudio exploratorio en tres escuelas secundarias públicas de Barcelona

A nivel autonómico, en Cataluña, la Ley 17/2015 (Ley de igualdad efectiva de mujeres y hombres 17/2015, de 21 de julio, 2015) hace referencia a la coeducación como motor de la igualdad de género y propone una educación sexual que favorezca "la construcción de una sexualidad positiva, saludable, que respete la diversidad y evite todo tipo de prejuicios por razón de orientación sexual y afectiva" (p. 79001), y se insta a la formación en educación sexual al personal de los centros educativos, de los educadores en deporte y tiempo libre y de todas las personas implicadas en el proceso educativo. La Ley 11/2014 (Ley para garantizar los derechos de lesbianas, gays, bisexuales, transgénero e intersexuales y para erradicar la homofobia, la bifobia y la transfobia 11/2014, de 10 de octubre, 2014) regula las cuestiones relacionadas con el respeto a la diversidad sexual desde el punto de vista educativo, incluyendo la formación de madres, padres y docentes con el fin de evitar la discriminación por causas de orientación, identidad o expresión sexual del estudiantado o sus familias. La Ley 12/2009 (Ley de Educación 12/2009, de 10 de julio, 2009) garantiza la coeducación, respetando la igualdad de género en todos los ámbitos educativos. Finalmente, la Ley 5/2008 (Ley de los derechos de las mujeres a erradicar la violencia machista 5/2008, de 24 de abril, 2008) explica que el modelo coeducativo "valora indistintamente la experiencia, las aptitudes y la aportación social y cultural de las mujeres y los hombres, en igualdad de derechos, sin estereotipos sexistas y androcéntricos, ni actitudes discriminatorias" (p. 18) y propone la creación de políticas públicas que colaboren en la educación para la erradicación de la violencia machista.

Para hacer efectivo el cumplimiento de estas leyes, y de cada uno de los puntos que desarrollan, existen normativas, directrices y guías que permiten que las instituciones públicas pongan en marcha programas que garanticen el acceso a la educación sexual y afectiva. Entre estas instituciones se encuentran los organismos internacionales que ponen a la salud sexual, desde un enfoque integral, en la agenda de los derechos humanos y el desarrollo sostenible con iniciativas como los Objetivos de Desarrollo Sostenible (Organización de las Naciones Unidas, 2015), en los cuales se hace continua referencia a la necesidad del acceso igualitario a los Derechos Sexuales y Reproductivos en todos los países, a través de políticas públicas que favorezcan, sobre todo, a las poblaciones en riesgo de vulnerabilidad.

\section{Objetivos}

El principal objetivo de este estudio fue analizar la implementación de la educación sexual en la enseñanza secundaria obligatoria. Para esto se definieron tres objetivos específicos: conocer la percepción de los docentes y estudiantes sobre la educación sexual en la escuela; conocer qué tipo de acciones de educación sexual se implementan en la escuela según docentes y estudiantes y la valoración que hacen; y recoger las demandas y propuestas de mejora que expresan docentes y estudiantes con relación a la educación sexual en la escuela.

\section{Método}

Se investigó desde un enfoque cualitativo que, como explican Rodríguez-Gómez et al. (1996), da la posibilidad de trabajar sobre las particularidades de cada una de las personas que participaron a partir de un método que "confía en las expresiones subjetivas, escrita y verbal, de los significados dados por los propios sujetos estudiados" (p. 64). 


\subsection{Participantes}

Para llevar adelante las entrevistas se seleccionaron tres institutos públicos de educación secundaria de la ciudad de Barcelona ubicadas en los barrios de La Vall d'Hebron, Les Roquetes y Pedralbes. Las dos primeras escuelas fueron contactadas gracias a la colaboración de la Associació de Planificació Familiar de Catalunya i Balears, con la tercera escuela se estableció relación a través de una docente de la Universidad de Barcelona.

Se entrevistó a una tutora y un tutor de 3 er $^{\text {er }}$ curso de Educación Secundaria Obligatoria (ESO) y una coordinadora de actividades extraescolares de 2.․ ciclo de ESO.

Para seleccionar a las y los estudiantes se definieron los siguientes criterios:

- Que estuvieran motivadas y motivados para participar

- Que tuvieran buena capacidad de comunicación

- Que no formaran parte del mismo grupo de pertenencia

- Que existiera paridad de género en el grupo

La participación del alumnado en el estudio fue completamente voluntaria, por lo que no se pudo mantener la paridad de género. Finalmente, participaron un total de doce estudiantes de segundo ciclo de ESO, de los cuales nueve eran mujeres (6 de $3 .{ }^{\text {er }}$ curso y 3 de 4. año) y tres varones de $3 .{ }^{\text {er }}$ curso.

\subsection{Recogida de datos}

En cada escuela se realizó una entrevista grupal al alumnado, y una entrevista individual a cada docente.

Las guías de preguntas de las entrevistas se organizaron según las dimensiones que se pretendían analizar: a) conceptualización de la Educación Afectiva y Sexual, b) participación en actividades o formaciones, c) demandas y propuestas de las y los estudiantes y d) percepción de las y los docentes sobre las necesidades del estudiantado.

\subsection{Análisis de datos}

Las entrevistas fueron registradas en audio y posteriormente transcritas. El análisis de contenido se realizó mediante el software informático Atlas.ti. La información se organizó a través de un sistema de códigos según dimensiones, categorías e informantes, respondiendo a los objetivos del estudio.

\subsection{Aspectos éticos}

Todo el proceso de investigación se llevó a cabo respetando el Código de Buenas Prácticas de la Universidad de Barcelona (2019a). Durante cada una de las etapas se tuvieron en cuenta los principios de honestidad, rigor y responsabilidad. De la misma manera, y comprometida con los objetivos planteados en el Estatuto de la Universidad (Decreto por el que se aprueba el Estatuto de 
la Universidad de Barcelona 246/2003, de 8 de octubre. Parlamento de Cataluña, 2003), en que se pretende luchar contra las desigualdades sociales y favorecer la igualdad entre hombres y mujeres -utilizando los mismos términos que constan en dicho decreto-, esta investigación pretende ser un aporte al conocimiento en las materias de salud y educación.

Para la realización de las entrevistas se redactó un consentimiento informado en el que se explicaba, de manera detallada, el alcance del estudio y el compromiso de anonimato de sus participantes y con la devolución de los resultados para quienes estuviesen interesados en recibir las conclusiones del estudio.

Para la redacción de este estudio se tuvieron en cuenta los criterios de la Universidad de Barcelona (2019b), como el uso de los recursos inclusivos, que incluyan a todos los géneros, o las formas dobles para el uso de un lenguaje que potencie el enfoque con perspectiva de género y permita la representación igualitaria de hombres y mujeres en los textos académicos. Se consideraron las recomendaciones de lenguaje tales como los recursos inclusivos, en los que se alude a hombres y mujeres, y los recursos visibilizadores, que hacen visibles las formas femeninas, según los requerimientos del texto.

\section{Resultados}

Se presentan los resultados según las dimensiones analizadas: a) conceptualización de la educación sexual, b) acciones de educación afectiva y sexual en la escuela, c) necesidades de los estudiantes y d) propuestas de mejora.

\subsection{Conceptualización de la Educación Sexual}

Las respuestas de las y los docentes responden a una idea de la educación sexual que vaya más allá de la perspectiva preventiva, que pueda brindar herramientas y orientaciones a las y los jóvenes para su desarrollo personal y en sociedad.

Sin embargo, la primera asignatura con la que se la relaciona es la biología, considerando que hay una cantidad de contenidos que pueden abordarse desde esa perspectiva, dejando en segundo lugar otras, como Ética y Valores. En este sentido, se revela una postura preventiva, con relación a la salud física, pero también a la salud mental, mencionando temas como los noviazgos violentos, la identidad de género, y otros conflictos que surgen durante la adolescencia.

Yo lo definiría como dotar a los alumnos de unas competencias mínimas, unos conocimientos mínimos para que puedan, ellos, desarrollar su vida afectivo sexual de manera sana saludable. (Profesora, Roquetes)

Estamos hablando ahora también de roles de género, de educación para la aceptación de otros tipos de sexualidades, de las relaciones de poder, las relaciones abusivas, todo ese tipo de cosas. Todo eso es lo que se tiene que tratar. (Tutor, Vall d'Hebron)

Las y los estudiantes presentan algunas dificultades en el momento definir el concepto de Educación Afectiva y Sexual, y lo relacionan directamente con la práctica de las relaciones sexuales, y derivado de eso, la prevención de las enfermedades y los embarazos no deseados. En una segunda lectura, coinciden con sus docentes en relacionarla con los contenidos sobre prevención de las violencias: 
Implementación de la Educación Afectiva y Sexual. Un estudio exploratorio en tres escuelas secundarias públicas de

Y que tenemos que pensar bien lo que hacemos y con quien, porque después te arrepientes mucho. (Estudiante mujer, Vall d'Hebron)

También que no te sientas obligado tampoco a hacer nada que no quieras, por la presión social y eso. Como adolescentes, sobre todo. (Estudiante mujer, Pedralbes)

\subsection{Acciones de Educación Afectivo Sexual en la ESO}

El profesorado describe algunas de las actividades realizadas, aunque desconocen la formación previa del estudiantado, especialmente en otros niveles de educación y en otros espacios como los de salud o educación no formal:

Lo que cuesta mucho es hacer un catálogo de todas las intervenciones que se hacen desde las diferentes materias. (...) porque incluso cada año depende del profesor se hacen unas cosas u otras. Hay profesores que les interesan más estos temas, otros menos. Entonces es difícil, es difícil tener una especie de esquema con todas las intervenciones. Pero bueno, hay algunas que se asegura que se hacen para todos los alumnos desde tutoría. (Tutor, Vall d’Hebron)

Según mencionan, existen diferentes espacios en los que, de manera intermitente o a demanda, se trabajan los contenidos de la EAS: las tutorías, la asignatura de biología, el programa Salut i Escola - con una enfermera que realiza visitas y tiene un espacio abierto de consultas - las visitas de profesionales de diferentes servicios de salud y para jóvenes, y otras asignaturas:

La [educación] sexual creo que las de ciencias es por ley que dan estos temas. En tutoría también las abarcamos, evidentemente. Pero creo que es que podrían ser extrapolables a cualquier otro tipo de asignatura. Yo, por ejemplo, soy profesora de inglés y la parte más afectiva intento tratarla en las asignaturas, la parte emocional, desde mi propia asignatura o mi propio ámbito de tutoría. (...) La parte sexual, nos vienen a hacer alguna charla desde el PIJ, que es el punto joven. (Tutora, Pedralbes)

Las y los estudiantes recuerdan algunas intervenciones en las que participaron, y hacen diferentes valoraciones según el interés que les provocaron. Explican que son intervenciones puntuales y esporádicas:

-Sí, en sexto vino una sexóloga. -Le pusimos un preservativo a una barra de pegamento... y ya está. Como éramos más niños nos lo tomamos un poquito a cachondeo, lo veíamos como un juego, casi. -Un poco de tabú también, porque como en la primaria, nada... En sexto tocamos un poco el tema de la reproducción. $-Y$ en la ESO, este año hicimos un trabajo sobre las enfermedades de transmisión sexual y vinieron a hacer un par de charlas, explicaron los métodos anticonceptivos. Cómo es el sexo en sí, el acto y ya está. -Más que actividad era como una exposición. Pusieron un Power Point y nos explicaron y nos hacían preguntas. (Estudiantes, Pedralbes)

Es que el primer taller estuvo bien, porque era la primera vez, pero en luego no hubo casi nada nuevo, fue lo de siempre, una repetición. (Estudiante, Vall d’Hebron)

Hacíamos actividades sobre, por ejemplo, lo que se hace en la discoteca y cuando te pasan cosas, empiezas a beber, y empiezas a tener relaciones sexuales con una persona, pues tienes que pensar en la protección y todo eso. Y saber las enfermedades que hay si no te pones condón. Fuimos al CAP a poner un condón. Y nos estuvieron hablando de las enfermedades. (Estudiante, Roquetes)

Con relación a la formación docente recibida, el profesorado explica que no ha recibido formación específica, a pesar de que existen algunas propuestas. Las que recuerdan son optativas, y entienden que no llegan a todos los docentes, consecuencia de la falta de tiempo: 
El año pasado sí que hicimos, teníamos, de la Agencia de Salud Pública, un dossier, y era sobre cómo se trataban muchas cosas: el tema de alimentación, un poco el tema de educación sexual, muchas cosas sobre autoestima. Pero es verdad que no recibimos, hablo en mi caso personal, ninguna formación ni como tutora ni como profesora. (Tutora, Pedralbes)

\subsection{Necesidades de los estudiantes}

Las y los docentes encuentran diferentes necesidades, que se desprenden de las temáticas que han podido trabajar en el aula, así como de la convivencia diaria con las y los estudiantes en la escuela. Se destacan el trabajo sobre las relaciones afectivas y sexuales sanas, el respeto a la diversidad sexual y de género, y el trabajo sobre las fuentes de información con las que cuentan las y los adolescentes:

A mí me preocupa la prisa que tienen por vivir, que necesitan tener una relación y necesitan que esa relación sea una relación, como ya de casi casarse. Tenemos parejas que llevan toda la ESO, y a veces un poco chocante en el sentido en que siento que se lo están imponiendo un poquito. (...) Tengo la sensación de que cada vez empiezan más pronto a experimentar, a jugar con consigo mismos y con tener relaciones sexuales. (Profesora, Roquetes)

Al mismo tiempo, destacan el importante papel de la formación entre iguales, impartida por aquellos y aquellas estudiantes que están más concienciados en cuestiones de género y LGTBI. Sin embargo, también señalan que, en otros casos, las estructuras familiares de las que provienen hacen necesaria la formación especializada:

Hay otros que vienen con unos estereotipos y unas pautas familiares (...) cosas sobre los celos, del estar celoso, que no es bueno, que es una forma de control, que no tienen que tomarlo como natural pero que tienen que ser conscientes que la otra persona tiene un derecho a vivir, a tener su espacio, de que no tienen que tener la contraseña del móvil y ellos te dicen 'que sí que la tengo que tener' y te lo razonan (...) así es como darte con una pared porque es que no llegas a ningún lado. (Profesora, Roquetes)

El estudiantado coincide en resaltar la necesidad el trabajo sobre las relaciones amorosas, mencionan la forma en que se relacionan y detectan "relaciones tóxicas" entre iguales. Además sostienen que ellos tienen poco conocimiento sobre estos temas y necesitan más información:

Es un tema súper extenso y nosotros, como adolescentes, no tenemos ni idea. Lo que sabemos nos llega de rebote. (Estudiante, Pedralbes)

Relaciones tóxicas, que hay muchísimas, y nunca nos han hablado, y hay gente que está en depresión por eso. Y nos han hablado de sexualidad, pero creo que tendrían que hablar de homosexualidad, de relaciones tóxicas, cosas así. (Estudiante, Vall d’Hebron)

\subsection{Propuestas de mejora}

El profesorado propone programas de Educación Afectiva y Sexual que puedan prolongarse en el tiempo y alcancen a todo el estudiantado, mientras que abarcan las diferentes problemáticas que surgen en los distintos contextos escolares:

La diversidad cultural (...) es una condición que tenemos que afrontar, que existe en las escuelas y es así. Y la diversidad cultural afecta a todos esos temas (...) A mí lo que me gustaría es tener un programa más fijo, que pueda decir, en primero hago esto, en segundo hago esto, pero es difícil. También falta formación del profesorado. Yo no creo que sea tan complicado, es una cuestión de voluntad de cambiar las cosas y de decir "esto es lo que quiero hacer y lo hago". (Tutor, Vall d'Hebron) 
También señalan la necesidad de brindar una EAS multidisciplinar, orientada desde diferentes puntos de vista, coordinada y que comience desde una edad más temprana, atravesando todos los niveles escolares, con el fin de abarcar los contenidos necesarios en cada etapa:

Pues yo creo que sería un poco la combinación de todo, charlas de gente más experta, formadores del Punto Joven, que igual no son expertos porque no tienen una carrera de años, pero sí que son mucho más cercanos y nosotros mismos de las mismas asignaturas. Si planteas un texto o una película, te puede salir tratar estas cosas seguro. Es que creo que debería ser algo como súper normalizado en las aulas, algo que deberíamos hacer todos desde todas las asignaturas que tenemos y talleres fuera, ir a centros de planificación familiar. (Profesora, Roquetes)

El estudiantado tiene una gran cantidad de propuestas y opiniones sobre cómo debería ser la educación que reciben, entre ellas, evitar la repetición de contenidos y poder desarrollar las temáticas con más profundidad y con dinámicas accesibles y atractivas, al mismo tiempo que se consideren sus intereses y necesidades:

Por ejemplo, en vez de hablar siempre de ponerse el condón, hablar de otra cosa, que sea relacionado con lo mismo, pero apartando un poco ese tema. (Estudiante Roquetes)

De los sentimientos, con tu pareja. (Estudiante, Roquetes)

El que venga, o la que venga, tiene que ser activo con los alumnos, porque si no los alumnos se duermen. No puedes estar una hora o dos dándole la chapa a 30 niños. (Estudiante Pedralbes)

Yo pondría unas actividades más didácticas. Porque todo el mundo dice que lo ha entendido y después no. (Estudiante, Vall d'Hebron)

\section{Discusión}

La propuesta de un modelo de educación sexual basada en una visión integral de la sexualidad está presente en el discurso de las y los entrevistados, tanto docentes como estudiantes, a pesar de que se continúa insistiendo en la prevención de enfermedades y embarazos como principal eje, y se apunta a una perspectiva desde las asignaturas de ciencias naturales.

Estudiantes y docentes encuentran una necesidad de trabajar sobre los contenidos de EAS, pero encuentran diferentes dificultades, como la falta de formación docente. Según explica SidaStudi (2018) en el ámbito educativo se deja la EAS a cargo del profesorado, esto se hace "sin apoyo y sin formación, la posibilidad de incorporarla de manera transversal en sus asignaturas o en las horas de tutoría, lo que en la práctica se queda en tierra de nadie" (p. 14). La falta de tiempo y recursos es una constante en el discurso docente, en el Plan de Salud Afectiva y Sexual (PSAS) se menciona que el profesorado considera su formación en la materia como insuficiente (Jané et al., 2014).

Otro aspecto que se resalta en el estudio es la valoración de los estudiantes con relación al tipo de intervenciones que se realizan, reclamando una mayor participación en las actividades y la innovación en los recursos y las temáticas. Este tipo de observaciones están presentes en investigaciones como la de Buenas Prácticas Pedagógicas en Educación Sexual Integral (Faur et al., 2018), desarrollado por UNICEF en Argentina:

Demandan espacios concretos donde la ESI sea una realidad tangible y donde la modalidad no sea tradicional, porque lo que demandan son espacios de conversación, espacios para reflexionar, para problematizar, para hacerse preguntas, para escuchar varias respuestas, para pensar. (p.118) 
Haciendo foco en los contenidos, no solo se menciona la nivelación por edades, sino también la adecuación a los diferentes contextos escolares, considerando la diversidad cultural y social y posibles desigualdades entre hombres y mujeres. Las situaciones de vulnerabilidad pueden acrecentarse en aquellos sitios donde la presencia del machismo en la cultura es más fuerte (Jané et al., 2014). El trabajo entre los distintos actores en la escuela: equipo docente, estudiantes y familia, puede permitir la identificación de situaciones de riesgo (Fundación Mujeres (UNED), 2004), mientras que el acceso a una educación sexual integral y transversal permite a los estudiantes reflexionar y reconocer los modelos normativos de género (Venegas, 2010). En la investigación llevada a cabo para la realización del PSAS (Jané et al., 2014) se hace especial mención en trabajar la EAS desde la perspectiva de género:

[...] se propone trabajar desde la masculinidad y la feminidad, ya que muchas conductas de riesgo tienen que ver con la concepción de 'ser mujer': amor romántico, sumisión de la mujer a la pareja, autoestima y carencias afectivas, responsabilidad en la prevención del embarazo... ${ }^{2}$ (p. 84-85)

En el estudio, docentes y estudiantes mencionan que se facilita el trabajo sobre la prevención de actitudes homófobas y tránsfobas en aquellos contextos donde los estudiantes han estado en contacto con personas del colectivo LGTBI, esto coincide con el informe de la Federación Estatal de Lesbianas, Gays, Transexuales y Bisexuales (FELGTB), en el cual se especifica que este contacto favorece las actitudes positivas frente a la diversidad sexual (Generelo, 2016), sumado a actividades formativas transversales que trabajen este temática (Martxueta \& Etxeberria, 2014).

Las propuestas de mejora del profesorado y el estudiantado que se recogen en este breve estudio exploratorio coinciden con las orientaciones propuestas por diferentes evidencias. Estas apuntan al trabajo transversal, y a la formación específica en cuestiones de Educación Afectiva y Sexual, durante todas las etapas de la educación formal. En todas se destaca la necesidad de una educación sexual que apunte al desarrollo de una sociedad más justa e igualitaria, a través de la promoción de la salud sexual y reproductiva y la prevención de los embarazos no deseados, las enfermedades de transmisión sexual y las violencias de género.

Las dificultades recogidas ponen en evidencia la necesidad de unas políticas públicas que sostengan el desarrollo de planes integrales y multidisciplinarios que contemplen la educación sexual en la escuela secundaria, así como en otros espacios educativos, en los servicios sociales, de salud y de justicia. Todo esto, apuntando a la formación de los profesionales, la acción y la intervención considerando las características sociales y culturales de todos los actores. Solo de esta manera se puede avanzar en la garantía del cumplimiento de los Derechos Sexuales y Reproductivos, como Derechos Humanos.

${ }^{2}$ Traducción de la autora del catalán al castellano. 


\section{Referencias}

Agencia EFE. (2018, mayo 28). Sube un 14,8 \% las menores protegidas víctimas de violencia machista. https://www.efe.com/efe/espana/sociedad/sube-un-14-8-las-menores-protegidas-victimas-deviolencia-machista/10004-3629888

Agencia EFE. (2019, junio 13). Baja el uso del condón y las infecciones por transmisión sexual suben un 26\%. https://www.efe.com/efe/espana/sociedad/baja-el-uso-del-condon-y-las-infecciones-portransmision-sexual-suben-un-26/10004-3999649

Aldavert, S. (2019). Presentació de la Campanya TIRA PEL(S) DRET(S)! [Comunicado de prensa]. https://bit.ly/3eL5sTI

BZgA. Federal Centre for Health and Education. (2010). Standards for Sexuality Education in Europe: A framework for policy makers, educational and health authorities and specialists. https://www.bzgawhocc.de/fileadmin/user_upload/WHO_BZgA_Standards_English.pdf

Calvo, G., Sierra, J., \& Caparrós, E. (2018). Derechos humanos y educación afectivo-sexual en la adolescencia: el programa educativo Forma Joven. Educació Social. Revista d'intervenció socioeducativa, 68, 80-101.

https://www.raco.cat/index.php/EducacioSocial/article/view/330790/428768

Cartledge, S., \& Ryan, J. (1983). Sex and love: new thoughts on old contradictions. The Women's Press.

Companys, M. (2016). La educación afectiva y sexual: un derecho, una prioridad. Creación positiva; CooperAcció; Associació de planificació familiar de Catalunya i Balears.

Consejería de Educación y Ciencia, \& Instituto Andaluz de la Mujer. (1999). Educación Afectivo-Sexual en la Educación Primaria. Guía para el profesorado. Junta de Andalucía.

Decreto por el que se aprueba el Estatuto de la Universidad de Barcelona 246/2003, de 8 de octubre. Parlamento de Cataluña, Diari Oficial de la Generalitat de Catalunya 20376 (2003).

Direcció de Feminismes i LGTBI. (2017). Diversitat en curt. Recursos pedagògics per treballar la diversitat afectiva, sexual i de gènere. Ajuntament de Barcelona. http://hdl.handle.net/11703/113861

Faur, E., Lavari, M., Ludman, F., \& Fuentes, S. (2018). Escuelas que enseñan ESI. Un estudio sobre Buenas Prácticas pedagógicas en Educación Sexual Integral. UNICEF. Presidencia de la Nación.

Font, P., Banyeres, D., \& Pérez Calvo, R. (2006). Orientacions per a l'educació afectiva i sexual a segon cicle d'ESO. Generalitat de Catalunya. http://www.sexualitatsana.cat/wpcontent/uploads/2017/05/SalutiEscola.pdf

Foucault, M. (1977). Historia de la sexualidad I- La voluntad de saber (2. ${ }^{\text {a }}$ ed.). Siglo XXI Editores.

Fundación Mujeres (UNED). (2004). Proyecto Detecta: Estudio de investigación sobre el sexismo interiorizado presente en el sistema de creencias de la juventud y adolescencia de ambos sexos y su implicación en la prevención de la violencia de género en el contexto de pareja. http://www.fundacionmujeres.es/img/Document/14941/documento.pdf 
Implementación de la Educación Afectiva y Sexual. Un estudio exploratorio en tres escuelas secundarias públicas de Barcelona

Garaizabal, C. (2009). Debates feministas sobre la sexualidad. En Granada, treinta años después. Aquí y ahora. Jornadas Feministas Estatales (pp. 397-404). Federación Estatal de Organizaciones Feministas. http://www.feministas.org/IMG/pdf/Debates_feministas_sobre_la_sexualidad_Cristina_Garaizabal.pdf

Generelo, J. (2016). La Diversidad sexual y de Género en el sistema Educativo: ¿qué sabemos sobre ella? Indice: Revista de Estadística y Sociedad, 66, 29-32. http://www.revistaindice.com/numero66/p29.pdf

Instituto Nacional de Estadística. (2003). Encuesta de Salud y Hábitos Sexuales.

Instituto Nacional de Estadística. (2018). Conviction Statistics: Adults / Conviction Statistics: Minors Year 2017. https://www.ine.es/en/prensa/ec_am_2017_en.pdf

Jané, M., Amorós, P., Molina, M. C., Vidal, M. J., \& Mateu, A. (2014). Pla de Salut Afectiva i Sexual: Promoció i prevenció en la infància i l'adolescéncia, amb especial èmfasi en la població vulnerable. Agència de Salut Pública Generalitat de Catalunya. http://hdl.handle.net/11351/1277

Kirby, D. (2007). Emerging answers: research findings on programs to reduce teen pregnancy. The National Campaign to Prevent Teen Pregnancy.

Kirby, D., Alter, J. S., \& Scales, P. (1980). An analysis of US sex education programs and evaluation methods (Vol. 1). Mathtec.

Ley de Educación 12/2009, de 10 de julio, Boletín Oficial del Estado (2009).

Ley de igualdad efectiva de mujeres y hombres 17/2015, de 21 de julio, Boletín Oficial del Estado 78986 (2015).

Ley de los derechos de las mujeres a erradicar la violencia machista 5/2008, de 24 de abril, Boletín Oficial del Estado 25174 (2008).

Ley de salud sexual y reproductiva y de la interrupción del embarazo 2/2010, de 3 de marzo, Boletín Oficial del Estado. Gobierno de España (2010).

Ley para garantizar los derechos de lesbianas, gays, bisexuales, transgénero e intersexuales y para erradicar la homofobia, la bifobia y la transfobia 11/2014, de 10 de octubre, Boletín Oficial del Estado 94729 (2014).

Martxueta, A., \& Etxeberria, J. (2014). Claves para atender la diversidad afectivo-sexual en el contexto educativo desde un enfoque global escolar. Revista Española de Orientación y Psicopedagogía, 25(3), 121-128. https://doi.org/10.5944/reop.vol.25.num.3.2014.13862

Mateos, A. (2014). Violencia: Tolerancia Zero. Guía práctica para la prevención de la violencia en secundaria. Obra Social «La Caixa». https://fundacionlacaixa.org/documents/10280/68385/Guia_prevencion_secundaria+1.pdf

Mateos, A., Balsells, M. À., Molina, M. C., Fuentes-Peláez, N., Pastor, C., \& Amorós, P. (2014). Necesidades educativas para promover la salud afectiva y sexual en jóvenes en riesgo social. REIRE Revista d'Innovació i Recerca en Educació, 7(2), 14-27. https://doi.org/10.1344/reire2014.7.2722 
Implementación de la Educación Afectiva y Sexual. Un estudio exploratorio en tres escuelas secundarias públicas de Barcelona

Observatorio para la Igualdad de Oportunidades. (2008). Guía de Coeducación. Síntesis sobre la Educación para la lgualdad de Oportunidades entre Mujeres y Hombres.

Organización de las Naciones Unidas. (2015). Objetivos de Desarrollo Sostenible.

Organización Mundial de la Salud. (2006). Defining sexual health. https://www.who.int/teams/sexual-andreproductive-health-and-research/key-areas-of-work/sexual-health/defining-sexual-health

Organización Mundial de la Salud, \& Human Reproduction Programme. (2018). La salud sexual y su relación con la salud reproductiva: un enfoque operativo. Organización Mundial de la Salud. https://www.who.int/reproductivehealth/publications/sexual_health/sh-linkages-rh/es/

Plataforma de Acción de Beijing. (1995). Declaración y Plataforma de Acción de Beijing. https://www.unwomen.org/-

/media/headquarters/attachments/sections/csw/bpa_s_final_web.pdf?la=es\&vs=755

Rebollo, J., Generelo, J., \& Assiego, V. (2019). La cara oculta de la violencia hacia el colectivo LGTBI. Federación Estatal de Lesbianas, Gais, Trans y Bisexuales.

Resolución del Parlamento Europeo sobre salud sexual y reproductiva y los derechos en esta materia 2001/2128(INI), C 271 E/36 Diario de la Unión Europea 369 (2003).

Rodríguez-Gómez, G., Gil-Flores, J., \& García-Jimenez, E. (1996). Proceso y fases de la investigación cualitativa. En Metodología de la Investigación Cualitativa. Algibe.

SidaStudi. (2018). De infundir miedo a difundir placeres. Claves reflexivas para la Educación Sexual. http://www.codajic.org/node/3550

UNESCO. (2010). Orientaciones Técnicas Internacionales sobre Educación en Sexualidad. Un enfoque basado en evidencia orientado a escuelas, docentes y educadores de la salud. https://educacionsexual.mineduc.cl/docs/2010_UNESCO_OrientacionesEdSexualUnesco.pdf

UNESCO. (2014). Educación integral de la sexualidad: conceptos, enfoques y competencias. UNESCO Office Santiago and Regional Bureau for Education in Latin America and the Caribbean. https://unesdoc.unesco.org/ark:/48223/pf0000232800

UNESCO. (2015). La Educación Integral en Sexualidad. Una revisión global de evidencia, prácticas y lecciones aprendidas. http://www.unesco.org/new/fileadmin/MULTIMEDIA/FIELD/Santiago/pdf/educacionintegral-sexualidad.pdf

UNESCO. (2018a). Orientaciones técnicas internacionales sobre Educación en Sexualidad: Un enfoque basado en la evidencia. https://unesdoc.unesco.org/ark:/48223/pf0000265335

UNESCO. (2018b). Por qué es importante la educación integral en sexualidad. https://es.unesco.org/news/que-es-importante-educacion-integral-sexualidad

Universitat de Barcelona. (2019a). Codi de bones pràctiques en recerca. Publicacions i Edicions de la Universitat de Barcelona.

Universitat de Barcelona. (2019b). Uso no sexista del lenguaje. Serveis Lingüístics. 
Implementación de la Educación Afectiva y Sexual. Un estudio exploratorio en tres escuelas secundarias públicas de

Venegas, M. (2010). Educar las relaciones afectivosexuales, prevenir las diferentes formas de violencia de género. Trabajo Social Global, 1(2), 162-182.

https://revistaseug.ugr.es/index.php/tsg/article/view/909

Weeks, J. (1981). Sex, Politics and Society: The regulation of sexuality since 1800. Routledge.

Zemaitis, S. (2016). Pedagogías de la sexualidad. Antecedentes, conceptos e historia en el campo de la educación sexual de la juventud [Trabajo final integrador, Universidad Nacional de La Plata]. http://www.memoria.fahce.unlp.edu.ar/tesis/te.1218/te.1218.pdf 